\title{
Management of Acute Pancreatitis
}

\author{
Case Report
}

\author{
M.A. Boermeester \\ Department of Surgery, Academic Medical Center, Amsterdam, The Netherlands
}

\section{Key Words}

Acute pancreatitis - Treatment - Antibiotics - Pancreatic necrosis · Surgery $\cdot$ Acalculous cholecystitis

\section{Case Report}

A 65-year-old male was admitted to the emergency room with abdominal pain and shock. Unfortunately, the diagnosis of acute pancreatitis was not made initially, the moderately elevated serum amylase levels were misinterpreted, and the patient underwent an emergency laparotomy because of his deplorable condition. Peroperatively, acute pancreatitis with a firm pancreas and minimal necrosis was found, there were no gallstones, and drains were placed in the lesser sac. The Imrie score was 3 points. Laboratory results revealed no cholestasis. The gram staining of abdominal fluid was negative for bacteria. The patient rapidly developed SIRS, ARDS, and DIC.

\section{Question}

Which antibiotic prophylaxis would you prefer, if any?
A Imipenem/cilastatin
B Cefuroxime
C Selective bowel decontamination
D No prophylaxis

Audience's response $74 \%$

$3 \%$

$10 \%$

$13 \%$

\section{Comment}

Secondary infection of pancreatic necrosis is the major cause of late deaths from acute pancreatitis. A recent meta-analysis showed a significant reduction of mortality from acute pancreatitis with the use of antibiotic prophylaxis. However, the included randomized trials all investigated different antibiotic regimens. The choice of a prophylactic agent should be made using the following criteria: (a) an adequate therapeutic level in pancreatic tissue, (b) broad-spectrum coverage of gram-negative bacteria as well as coverage of anaerobic species and Staphylococcus aureus (Escherichia coli, Pseudomonas species, anaerobic species, and $S$. aureus account for more than $70 \%$ of all infected pancreatic necrosis), and (c) documented efficacy in a least one randomized clinical trial. Following these criteria, there is mainly a rationale for prophylactic use of imipenem. Whether antibiotic prophylaxis should be given based on risk profiles by prognostic scoring systems, such as the Ranson, Imrie, and Apache II scores, or only when necrosis is found by CT imaging remains unclear. It is of note that the clinical trials on which the use of antibiotic prophylaxis is based nowadays, mainly used pancreatic necrosis as an inclusion criterium.

\section{Case Report (Continued)}

Imipenem was indeed administered to this patient when a CT scan 1 week later revealed necrosis of the pancreatic tail. In following 2 weeks, his clinical condition improved, although he remained on

\begin{tabular}{ll}
\hline KARGER & ( ) 2002 S. Karger AG, Basel \\
Fax +4161306 1234 & 0253-4886/02/0192-0125\$18.50/0 \\
$\begin{array}{l}\text { E-Mail karger@karger.ch } \\
\text { www.karger.com }\end{array}$ & $\begin{array}{l}\text { Accessible online at: } \\
\text { www.karger.com/journals/dsu }\end{array}$
\end{tabular}

\author{
Dr. M.A. Boermeester, Surgeon \\ Academic Medical Center, Department of Surgery (G4-127) \\ Meibergdreef 9 \\ NL-1105 AZ Amsterdam (The Netherlands) \\ Tel. +31 20 5662666, Fax +3120 6914858, E-Mail m.a.boermeester@amc.uva.nl
}




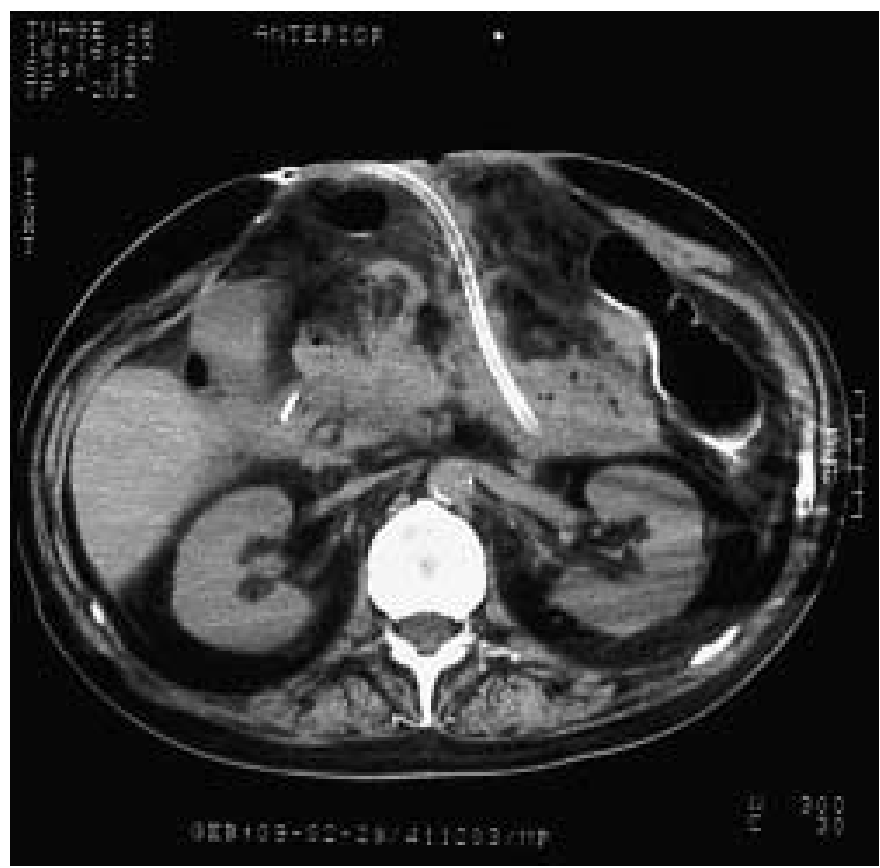

Fig. 1. Abdominal CT scan with fluid collections and air around the pancreatic tail which were drained percutaneously.

the ICU with minimal mechanical ventilator support and without inotropic medication. Adequate enteral feeding was given via an endoscopically placed nasojejunal tube. He returned to the hospital ward a month after admission. Repeated CT scan the same day showed fluid collections around the pancreatic tail, which were drained percutaneously (fig. 1), as well as tail necrosis. Gram staining of this drain fluid showed several gram-negative and gram-positive organisms. Later culture results would show these to be Staphylococcus epidermidis, Enterococcus sp., and Pseudomonas maltophilia.

\section{Question}

What would be your course of action in this patient with improving clinical condition but now infected necrosis?

\section{A No additional procedure \\ B Surgery}

Audience's response $34 \%$ $66 \%$

\section{Comment}

Management of infected pancreatic necrosis by percutaneous drainage cannot be advocated as a standard procedure. In carefully selected patients and in hospitals with extensive expertise in interventional radiology, percutaneous management of late infected necrosis may in some cases be an alternative. This may be effective provided the necrosis is semiliquid, large-diameter drains are used, and the procedure is combined with repeated lavage via the drain(s). In general, infected necrosis is managed by necrosectomy via laparotomy, although less invasive surgical procedures such as laparoscopy or drain tract endoscopy are used and reported in small series. Sterile necrosis as determined by CT-guided fine needle aspiration (FNA) and gram staining is managed primarily without surgical drainage. However, in patients with progressive organ failure despite ICU treatment or in patients with persistent organ failure after at least $72 \mathrm{~h}$ of ICU treatment, surgical drainage may be indicated. Important in this respect is to take into consideration that about $10 \%$ of the FNA results are false-negative.

\section{Case Report (Continued)}

In this patient, his improving clinical condition was reason to refrain from surgery. No additional procedure was performed. Two weeks later, in the 6th week after admission, he developed spiking fever $\left(>39^{\circ} \mathrm{C}\right)$. The drain production was persistent purulent $(50 \mathrm{ml} /$ day). An abdominal CT scan was performed and a large left lateral abscess was drained percutaneously. Although an initial improvement was seen, the spiking fever returned. On physical examination, there was abdominal distention, enteral feeding could not be increased beyond $20 \mathrm{ml} / \mathrm{h}$, but no signs of extrapancreatic organ failure. The leukocyte count was $22.3 \times 10^{9} / 1$. Abdominal CT imaging was repeated, which revealed a left lateral fluid collection (previous drain with its tip in this collection) and a large left-sided subphrenic fluid collection. There was a moderate, but homogeneous contrast enhancement of the pancreas.

\section{Question}

What is your next diagnostic/therapeutic step?

Audience's response

A Percutaneous drainage $35 \%$

B Laparotomy + closed drainage (Beger)

$11 \%$

C Laparotomy + open drainage (Bradley) $4 \%$

D Posterior drainage via 11 th or 12 th rib resection

E Minimal invasive drainage (drain tract endoscopy)

\section{Comment}

More than 6 weeks after the emergency laparotomy on day 1 and about 5 weeks after the development of pancreas necrosis and fluid collections, managed by percutaneous drainage successfully, is not the most optimal timing for a frontal surgical approach. Peripancreatic complications, such as abscesses, bleeding or necrosis of the transverse colon, are a valid reason for surgery. However, during the late stage of acute pancreatitis, infection is usually contained. Therefore, less invasive procedures 


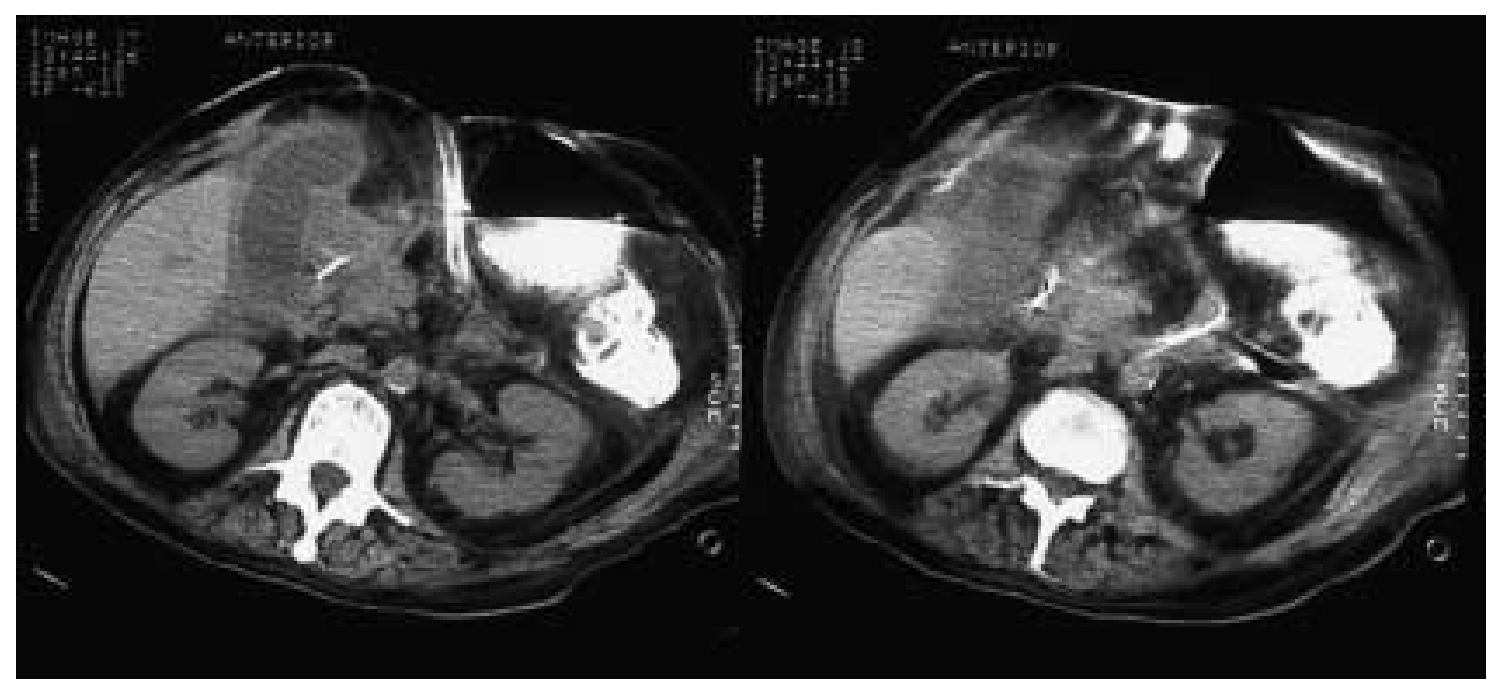

Fig. 2. Hydrops and thickened wall of the gallbladder with subhepatic fluid collection on CT imaging.

(percutaneous drainage, endoscopic drainage, flank drainage via rib resection or drain tract) are preferred.

\section{Case Report (Continued)}

Posterior drainage of both collections was performed via flank incision and 11th rib resection. For another 3 weeks, no fever was observed and he recovered progressively. During the 10th week after admission, the patient once again displayed high spiking fever, this time with hypotension (managed by fluid resuscitation; no ICU admission). A CT scan was performed, showing an abnormal gallbladder (fig. 2).

\section{Question}

Which therapy would you prefer?
A Cholecystectomy
B Percutaneous cholecystostomy

$\begin{aligned} & \text { Audience's } \\ & \text { response } \\ & 69 \% \\ & 31 \%\end{aligned}$

\section{Comment}

During the laparotomy on day 1 and all subsequent imaging, no gallstones were seen. The most likely diagnosis is an acute acalculous cholecystitis (AAC). The CT image is highly suspicious for acalculous gallbladder empyema. Acalculous cholecystitis is usually caused by decreased gallbladder motility and starvation in already ill patients after trauma, burns, surgery, prolonged use of TPN, or narcotics. AAC occurs in $0.2 \%$ of surgical ICU admissions and accounts for $5-10 \%$ of all cases of acute cholecystitis. Presenting symptoms and laboratory values were nonspecific. Therefore, a high index of suspicion is necessary to diagnose AAC in critically ill patients with sepsis. Ultrasonography and CT may aid in diagnosis. Prompt diagnosis is important because the clinical course is often fulminant, with gangrene or perforation. Mortality from AAC can be as high as $40 \%$. When the disease is present, prompt intervention with either percutaneous cholecystostomy or surgical management is indicated. To our opinion, percutaneous cholecystostomy is preferred in already critically ill patients, because of the high risk of biliary and nonbiliary complications during surgery. Either CT imaging or clinical deterioration of ICU patients may indicate gallbladder necrosis and/or perforation, in which case cholecystectomy is preferred.

\section{Case Report (Continued)}

Percutaneous cholecystostomy was performed promptly in our patient (fig. 3). In the end, because of clinical deterioration needing ICU treatment, a cholecystectomy had to be performed. During laparotomy a necrotic gallbladder was removed and a subhepatic abscess was drained. A subhepatic drain was placed, which demonstrated bile leakage after a few days.

\section{Question}

Which diagnostic/therapeutic step would you prefer? Audience's
A Surgery
B ERCP and stenting
C PTC drainage response

$40 \%$

\section{Comment}

The circumstances during which the cholecystectomy was performed were already difficult. Importantly, a rela- 


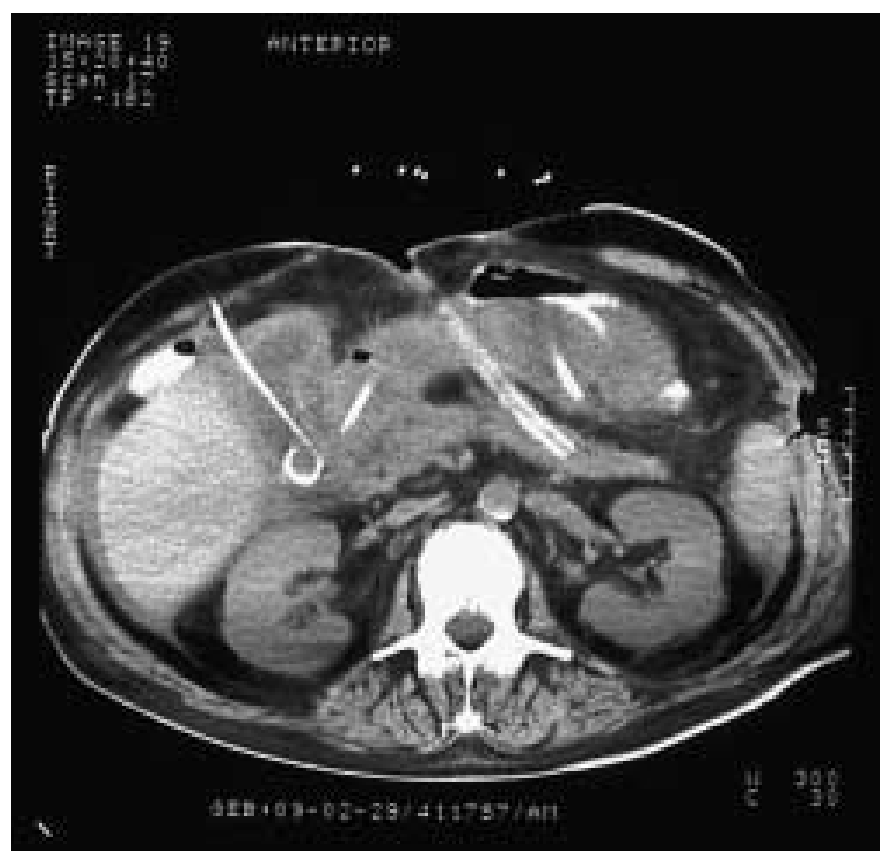

Fig. 3. CT scan after percutaneous cholecystostomy with pigtail drain. Large-diameter drain in pancreatic tail area still in situ.

parotomy should be avoided at this point because there is no information about the injury, since the anatomy of the biliary tract is not yet visualized. Relaparotomy for drainage is not necessary, since the leak already drains via the intra-abdominal drain. In addition, acute or subacute reconstructions have poor results and should not be per-

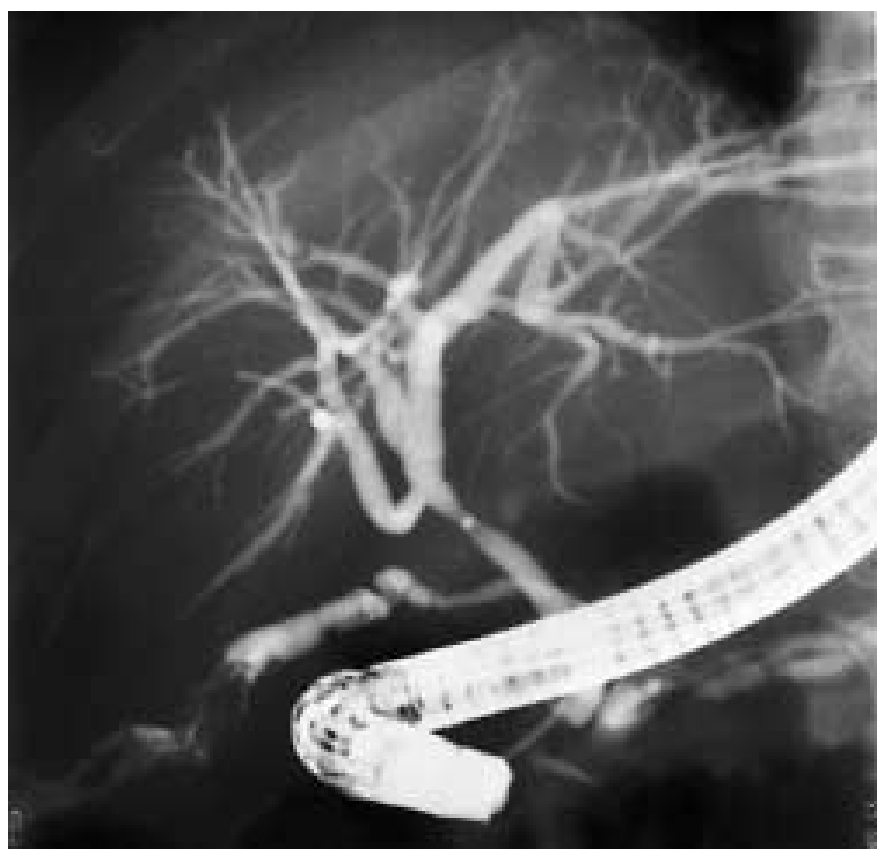

Fig. 4. ERCP showing cystic stump leakage after open cholecystectomy for a necrotic gallbladder.

formed. ERCP and PTC are both applicable, depending on local expertise.

\section{Case Report (Continued)}

ERCP revealed a leak from the cystic stump (fig. 4), which was managed by a CBD stent. The patient was discharged after 3 months and the stent was removed after 1 year without further problems. 